\title{
Research Article \\ Exact Solution of Impulse Response to a Class of Fractional Oscillators and Its Stability
}

\author{
Ming Li, ${ }^{1}$ S. C. Lim, ${ }^{2}$ and Shengyong Chen ${ }^{3}$ \\ ${ }^{1}$ School of Information Science and Technology, East China Normal University, no. 500, \\ Dong-Chuan Road, Shanghai 200241, China \\ 228 Farrer Road, \#05-01, Sutton Place, Singapore 268831 \\ ${ }^{3}$ College of Computer Science, Zhejiang University of Technology, Hangzhou 310023, China \\ Correspondence should be addressed to Ming Li, ming_lihk@yahoo.com
}

Received 18 August 2010; Accepted 15 September 2010

Academic Editor: Cristian Toma

Copyright (C) 2011 Ming Li et al. This is an open access article distributed under the Creative Commons Attribution License, which permits unrestricted use, distribution, and reproduction in any medium, provided the original work is properly cited.

\begin{abstract}
Oscillator of single-degree-freedom is a typical model in system analysis. Oscillations resulted from differential equations with fractional order attract the interests of researchers since such a type of oscillations may appear dramatic behaviors in system responses. However, a solution to the impulse response of a class of fractional oscillators studied in this paper remains unknown in the field. In this paper, we propose the solution in the closed form to the impulse response of the class of fractional oscillators. Based on it, we reveal the stability behavior of this class of fractional oscillators as follows. A fractional oscillator in this class may be strictly stable, nonstable, or marginally stable, depending on the ranges of its fractional order.
\end{abstract}

\section{Introduction}

Fractional systems gain increasing attention in applied sciences, ranging from mechanical engineering to electrical engineering, see, for example, [1-11]. Recall that stability is an essential property of systems, see for example [12], for the stability of conventional systems of integer order and [13-15] for fractional systems.

One of the typical models used in system analysis is the oscillator of single-degreefreedom [16]. It is given by

$$
m \frac{d^{2} Y(t)}{d t^{2}}+c \frac{d Y(t)}{d t}+k Y(t)=e(t)
$$


where $m>0$ is the mass, $c$ the damping constant, $k>0$ the stiffness and $e(t)$ the forcing function. Let $2 b=c / m \geq 0$ and $\omega_{0}=\sqrt{\mathrm{k} / \mathrm{m}}$. Then, we rewrite the above by

$$
\frac{d^{2} Y(t)}{d t^{2}}+2 b \frac{d Y(t)}{d t}+\omega_{0}^{2} Y(t)=e(t)
$$

The parameter $b$ is called damping coefficient and $\omega_{0}$ is inherent frequency.

Let $g(t)$ be the impulse response to the above equation. It is the solution to (1.2) for $e(t)=\delta(t)$ (the Dirac delta function) with zero initial conditions and is given by

$$
g(t)=\frac{1}{\omega} e^{-b t} \sin (\omega t)
$$

where $\omega=\sqrt{\omega_{0}^{2}-b^{2}}$ is angular frequency. Equation (1.3) implies a damped oscillation.

In the case of $b=0$, that is, the zero damping, (1.2) reduces to

$$
\left(\frac{d^{2}}{d t^{2}}+\omega_{0}^{2}\right) Y(t)=e(t), \quad \omega_{0}>0
$$

The impulse response to the above system is

$$
g(t)=\frac{1}{\omega_{0}} \sin \left(\omega_{0} t\right)
$$

which corresponds to a free oscillation.

Recently, research on fractional oscillators has attracted considerable interests, see for example, [17-22]. In the stability analysis of fractional oscillators, the authors in [17-19] studied a class of fractional oscillators expressed by

$$
\frac{d^{2-\varepsilon}}{d t^{2-\varepsilon}}+\omega_{0}^{2} x(t)=e(t), \quad 0<\varepsilon<1
$$

They concluded that the above oscillator may be strictly stable as if it is a damped oscillator. In this paper, we focus on another class of fractional oscillators that were first introduced by Lim and Muniandy [23]. It satisfies

$$
\left(\frac{d^{2}}{d t^{2}}+\omega_{0}^{2}\right)^{\beta} x(t)=e(t), \quad \beta>0 .
$$

The solutions to (1.7) for $e(t)$ being a white noise in time domain and frequency domain are obtained in [21], which are further extended to the oscillator with two fractional indexes in $[22,24,25]$. However, its stability remains an unsolved issue. This paper shows that this type of fractional oscillator is strictly stable when $0<\beta<1$, nonstable when $\beta>1$, and marginally stable for $\beta=1$. 
The remainder of the paper is organized as follows. In Section 2, the impulse response of the fractional system (1.7) in the closed form is proposed. Stability analysis is given in Section 3. Discussions and conclusions are in Section 4.

\section{Impulse Response}

For $t>0$ and $v>0$, denote by $D_{t}^{v}$ the fractional derivative of Caputo type [26] defined by

$$
D_{t}^{v} f(t)=\frac{1}{\Gamma(n-v)} \int_{0}^{t} \frac{f^{(n)}(u) d u}{(t-u)^{v-n+1}}, \quad n-1 \leq \alpha \leq n
$$

where $\Gamma$ is the Gamma function. For simplicity, we write ${ }_{0} D_{t}^{v}$ by $D^{v}$ below. One can regard $\left(D^{2}+\omega_{0}^{2}\right)^{\beta}$ as a shifted fractional derivative of $D^{2 v}$. By taking the binomial expansion, one gets

$$
\left(D^{2}+\omega_{0}^{2}\right)^{\beta}=\sum_{n=0}^{\infty}(\beta)_{n} \omega_{0}^{2 n} D^{2(\beta-n)},
$$

where $(\beta)_{m}$ is the Pochhammer symbol, that is,

$$
(\beta)_{m}=\frac{\beta !}{(\beta-m) !}
$$

The fractional oscillator satisfying $\left(D^{2}+\omega_{0}^{2}\right)^{\beta} x(t)=e(t), \quad \beta>0$ has the impulse response function $h(t)$, which is the solution to $\left(D^{2}+\omega_{0}^{2}\right)^{\beta} h(t)=\delta(t)$. Denote the Laplace transform of $h(t)$ by $H(s)$. Then, we have

$$
H(s)=\mathrm{L}[h(t)]=\frac{1}{\left(s^{2}+\omega_{0}^{2}\right)^{\beta}}=\frac{1}{\omega_{0}^{2 \beta}\left(1+s^{2} / \omega_{0}^{2}\right)^{\beta}},
$$

where $\mathrm{L}$ is the operator of the Laplace transform. Expanding the right side of the above using the binomial series yields

$$
\frac{1}{\omega_{0}^{2 \beta}\left(1+s^{2} / \omega_{0}^{2}\right)^{\beta}}=\frac{1}{\omega_{0}^{2 \beta}} \sum_{m=0}^{\infty} \frac{(\beta)_{m}}{m !}(-1)^{m} s^{m}, \quad\left|\frac{s}{\omega_{0}}\right|<1 .
$$

Therefore, the impulse response to (1.7) is given by

$$
h(t)=\frac{1}{\omega_{0}^{2 \beta}} \mathrm{L}^{-1}\left[\sum_{m=0}^{\infty} \frac{(\beta)_{m}}{m !}(-1)^{m} s^{m}\right]=\frac{1}{\omega_{0}^{2 \beta}} \sum_{m=0}^{\infty} \frac{(\beta)_{m}}{m !}(-1)^{m} \delta^{(m)}(t), \quad t \geq 0,
$$

where $\mathrm{L}^{-1}$ is the operator of the inverse Laplace transform. 
Another form of $h(t)$, which may be more convenient for the stability analysis, is expressed below. Considering the Laplace transform pair given in [27], we have

$$
\mathrm{L}^{-1}\left[\frac{1}{\left(s^{2}+\omega_{0}^{2}\right)^{\beta}}\right]=\frac{\sqrt{\pi}}{\Gamma(\beta)\left(2 \omega_{0}\right)^{\beta-1 / 2}} t^{\beta-1 / 2} J_{\beta-1 / 2}\left(\omega_{0} t\right),
$$

where $J_{\beta-1 / 2}\left(\omega_{0} t\right)$ is the Bessel function of the first kind of order $\beta-1 / 2$. Therefore, we have the impulse response given by

$$
h(t) \triangleq h(t ; \beta)=\frac{\sqrt{\pi}}{\Gamma(\beta)\left(2 \omega_{0}\right)^{\beta-1 / 2}} t^{\beta-1 / 2} J_{\beta-1 / 2}\left(\omega_{0} t\right), \quad \beta>0, t \geq 0
$$

\section{Stability Analysis}

The above discussion allows us to obtain the following results concerning the stability of the fractional oscillator under consideration. Before we discuss these stability properties, we first recall the criteria of stability based on the principle of bounded-input and bounded-output (BIBO). A system is said to be stable if

$$
\int_{0}^{\infty}|h(t)| d t=\text { constant }
$$

which implies $\lim _{t \rightarrow \infty} h(t)=0$ and poles of $L[h(t)]$ are located on the left-hand portion of the $s$-plane. A system is said to be nonstable if $h(t)$ is increasing, and accordingly poles of $L[h(t)]$ are located on the right-hand portion of the s-plane. One says that a system is neutral if poles of $L[h(t)]$ are on the complex $j \omega$-axis $[28,29]$.

Note that [27]

$$
J_{v}(t)=\frac{(t / 2)^{v}}{\Gamma(v+1 / 2) \Gamma(1 / 2)} \int_{-1}^{1}\left(1-u^{2}\right)^{v-1 / 2} \cos (t u) d u, \quad \operatorname{Re} v>-1 / 2
$$

Thus, according to (3.2) for $v=1 / 2$ and considering (2.8) for $\beta=1, h(t ; \beta)$ in (2.8) reduces to the simple case with impulse response corresponds to the free oscillator. That is,

$$
\left.h(t ; \beta)\right|_{\beta=1}=\frac{\sin \omega_{0} t}{\omega_{0}}
$$

This leads to the following remark.

Remark 3.1. $h(t ; \beta)$ reduces to the impulse response to the ordinary oscillator for $\beta=1$. Figure 1 indicates the plot of $h(t ; 1)$ for $\omega_{0}=1$. The ordinary oscillator is neutral. 


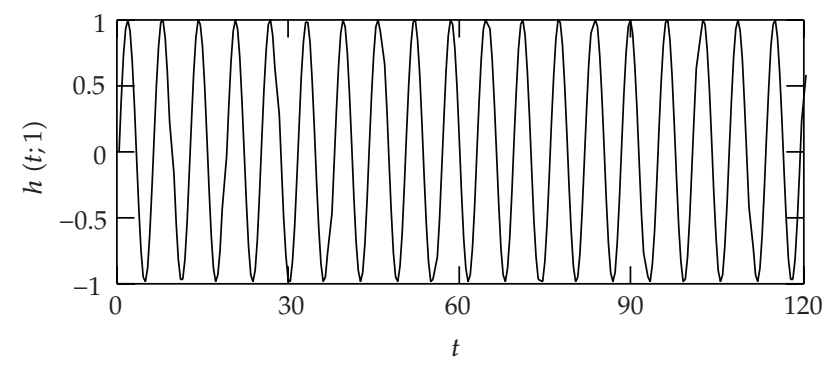

Figure 1: Plot of the impulse response of the ordinary oscillator.

One notes that $h(t ; \beta)$ is unbounded if $\beta>1$. As a matter of fact, from (3.2), we have

$$
\frac{-(t / 2)^{v}}{\Gamma(v+1 / 2) \Gamma(1 / 2)} \int_{-1}^{1}\left(1-u^{2}\right)^{v-1 / 2} d u \leq J_{v}(t) \leq \frac{(t / 2)^{v}}{\Gamma(v+1 / 2) \Gamma(1 / 2)} \int_{-1}^{1}\left(1-u^{2}\right)^{v-1 / 2} d u
$$

Since $\beta>1$ implies $v>1 / 2$, one immediately sees that both the right side and the left one on the above expression are, respectively, unbounded as $t \rightarrow \infty$. Figure 2 shows the oscillations for various values of $\beta$ for $w_{0}=1$. Thus, we have the following remark.

Remark 3.2. For $\beta>1$, the fractional oscillator (1.7) is non stable.

The other interesting thing is that the $h(t ; \beta)$ becomes an oscillation with decreasing amplitude if $0<\beta<1$. In fact,

$$
J_{v}(t)=\sum_{m=0}^{\infty} \frac{(-1)^{m}}{m ! \Gamma(m+v+1)}\left(\frac{t}{2}\right)^{2 m+v}
$$

Thus,

$$
J_{v}(t) \sim \frac{1}{\sqrt{t}} \text { for } t \longrightarrow \infty
$$

Consequently, we have

$$
\lim _{t \rightarrow \infty} h(t ; \beta)=\lim _{t \rightarrow \infty} \frac{\sqrt{\pi}}{\Gamma(\beta)\left(2 \omega_{0}\right)^{\beta-1 / 2}} t^{\beta-1 / 2} J_{\beta-1 / 2}\left(\omega_{0} t\right)=0, \quad 0<\beta<1
$$

which implies that the poles of $\mathrm{L}[h(t ; \beta)]$ for $0<\beta<1$ are located on the left of the $s$-plane. Therefore, the following remark is an obvious consequence. Figure 3 gives plots of $h(t ; \beta)$ for several values of $\beta$ for $\omega_{0}=1$.

Remark 3.3. The system (1.7) is strictly stable for $0<\beta<1$.

The stability of the fractional oscillators expressed by (1.7) is summarized in Table 1. 


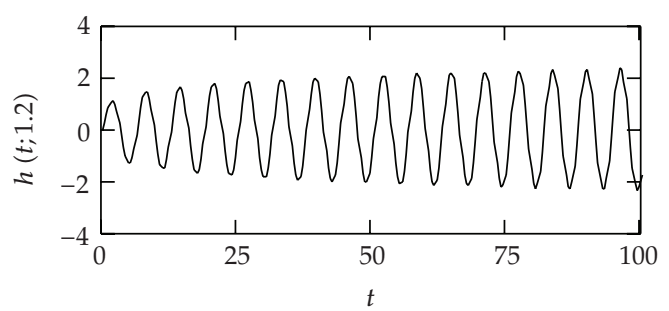

(a)

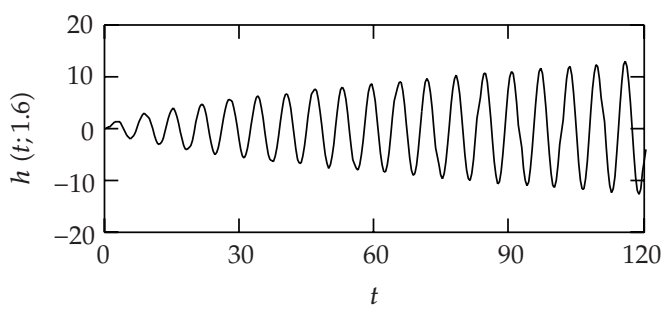

(c)

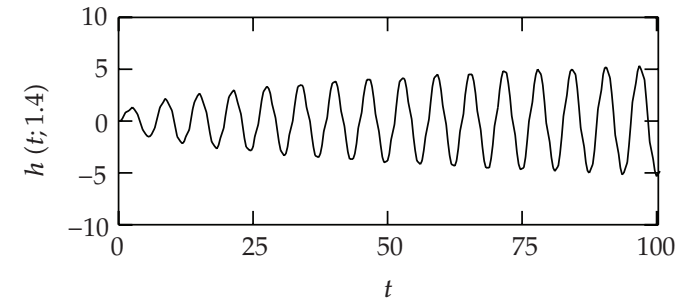

(b)

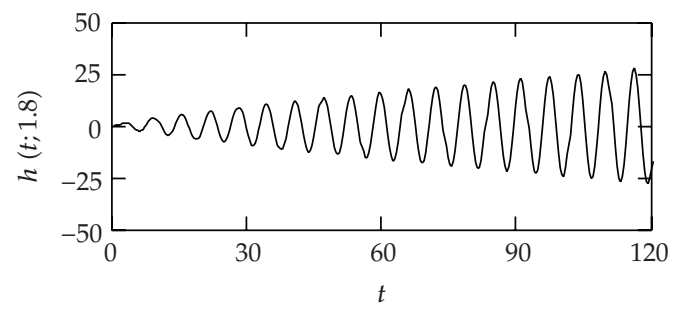

(d)

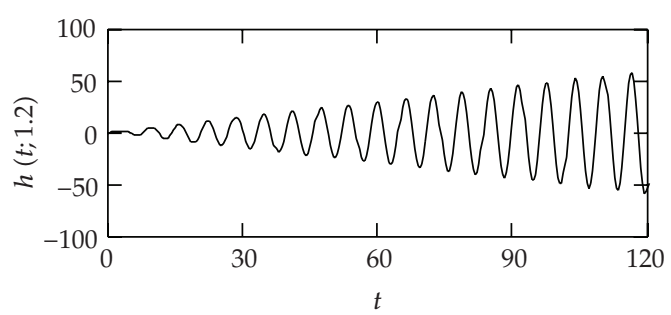

(e)

Figure 2: Increasing oscillations. (a) $\beta=1.2$. (b) $\beta=1.4$. (c) $\beta=1$.6. (d) $\beta=1.8$. (e) $\beta=1.2$.

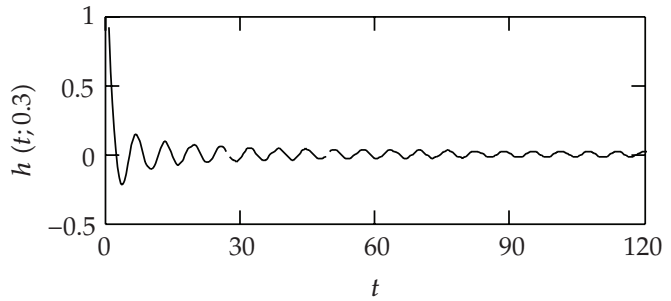

(a)

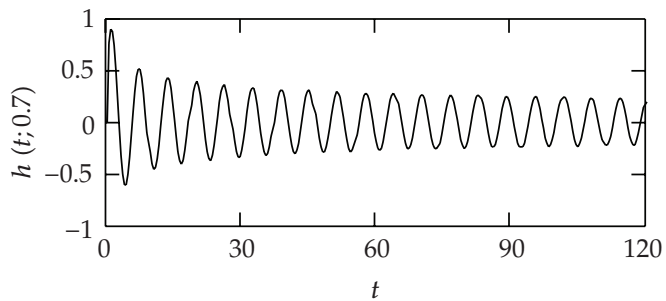

(c)

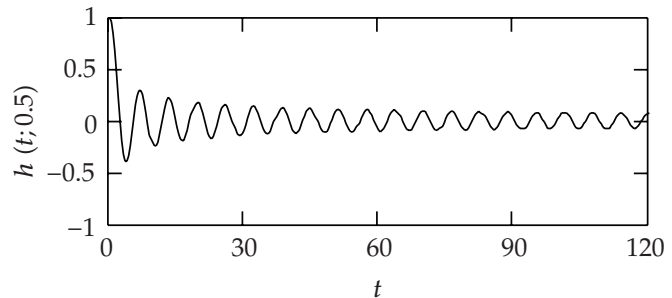

(b)

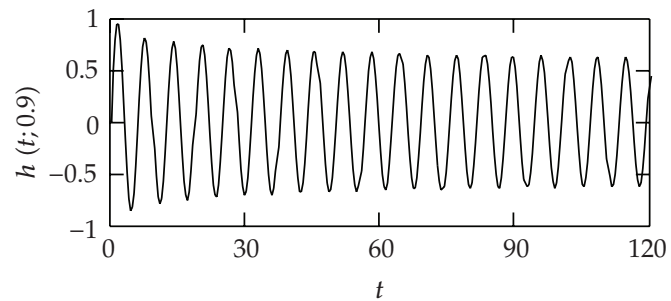

(d)

Figure 3: Oscillations. (a) $\beta=0.3$ (b) $\beta=0.5$ (c) $\beta=0.7$ (d) $\beta=0.9$. 
Table 1: Stability performances of (1.7).

\begin{tabular}{lc}
\hline Value of $\beta$ & Type of stability \\
\hline $0<\beta<1$ & Strictly stable \\
$\beta>1$ & Non stable \\
$\beta=1$ & Neutral \\
\hline
\end{tabular}

\section{Discussions And Conclusions}

As previously noted in [25], a fractal time series can be considered as a solution to a fractional differential equation driven by a white noise. Thus, there may be research niche for other series, for example, those discussed in [30-43]. In this paper, we have presented two forms, that is, (2.6) and (2.8), of the impulse response to the fractional system expressed by (1.7). Such a type of fractional oscillators has dramatic performances in its stability. We have revealed that the system with (1.7) contains three subclasses of oscillators. Ordinary free oscillators are a special case of (1.7) for $\beta=1$. It corresponds to fractional oscillators of strictly stable for $0<\beta<1$ and non stable if $\beta>1$. Note that there is no damping term in the discussed oscillators in form. However, in the case of $0<\beta<1$, they are strictly stable, as though they were ordinary oscillators equipped with a certain damping. On the other side, for $\beta>1$, they are non stable.

\section{Acknowledgments}

This work was partly supported by the National Natural Science Foundation of China (NSFC) under the Project Grant nos. 60573125, 60873264, 61070214, 60870002, 60703112, and 60974001, the 973 plan under the Project no. 2011CB302800, NCET, and the Science and Technology Department of Zhejiang Province (2009C21008, 2010R10006, 2010C33095, Y1090592). The discussions with Dr. Yang-Quan Chen of the Utah State University are helpful.

\section{References}

[1] M. D. Ortigueira and A. G. Batista, "On the relation between the fractional Brownian motion and the fractional derivatives," Physics Letters A, vol. 372, no. 7, pp. 958-968, 2008.

[2] M. D. Ortigueira, "An introduction to the fractional continuous-time linear systems: the 21st century systems," IEEE Circuits and Systems Magazine, vol. 8, no. 3, pp. 19-26, 2008.

[3] Y. Luo and Y. Chen, "Fractional order [proportional derivative] controller for a class of fractional order systems," Automatica, vol. 45, no. 10, pp. 2446-2450, 2009.

[4] Y. Q. Chen and K. L. Moore, "Discretization schemes for fractional-order differentiators and integrators," IEEE Transactions on Circuits and Systems I, vol. 49, no. 3, pp. 363-367, 2002.

[5] J. A. Tenreiro Machado, M. F. Silva, R. S. Barbosa et al., "Some applications of fractional calculus in engineering," Mathematical Problems in Engineering, vol. 2010, Article ID 639801, 34 pages, 2010.

[6] O. P. Agrawal, "Solution for a fractional diffusion-wave equation defined in a bounded domain," Nonlinear Dynamics, vol. 29, no. 1-4, pp. 145-155, 2002.

[7] I. Podlubny, I. Petráš, B. M. Vinagre, P. O’Leary, and L. Dorčák, “Analogue realizations of fractionalorder controllers," Nonlinear Dynamics, vol. 29, no. 1-4, pp. 281-296, 2002.

[8] C. H. Eab and S. C. Lim, "Path integral representation of fractional harmonic oscillator," Physica A, vol. 371, no. 2, pp. 303-316, 2006.

[9] C. H. Eab and S. C. Lim, "Fractional generalized Langevin equation approach to single-file diffusion," Physica A, vol. 389, no. 13, pp. 2510-2521, 2010. 
[10] C.-C. Tseng and S.-L. Lee, "Digital IIR integrator design using recursive Romberg integration rule and fractional sample delay," Signal Processing, vol. 88, no. 9, pp. 2222-2233, 2008.

[11] V. Lakshmikantham, S. Leela, and J. Vasundhara Devi, Theory of Fractional Dynamic Systems, Cambridge Scientific Publishers, Cambridge, UK, 2009.

[12] C. M. Harris, Ed., Shock and Vibration Handbook, McGraw-Hill, New York, NY, USA, 4th edition, 1995.

[13] Y. Chen and K. L. Moore, "Analytical stability bound for a class of delayed fractional-order dynamic systems," Nonlinear Dynamics, vol. 29, no. 1-4, pp. 191-200, 2002.

[14] H.-S. Ahn and Y. Chen, "Necessary and sufficient stability condition of fractional-order interval linear systems," Automatica, vol. 44, no. 11, pp. 2985-2988, 2008.

[15] D. Qian, C. Li, R. P. Agarwal, and P. J. Y. Wong, "Stability analysis of fractional differential system with Riemann-Liouville derivative," Mathematical and Computer Modelling, vol. 52, no. 5-6, pp. 862874, 2010.

[16] S. S. Antman, J. E. Marsden, and L. Sirovich, Eds., Applied Delay Differential Equations, Springer, New York, NY, USA, 2009.

[17] B. N. N. Achar, J. W. Hanneken, T. Enck, and T. Clarke, "Dynamics of the fractional oscillator," Physica A, vol. 297, no. 3-4, pp. 361-367, 2001.

[18] B. N. N. Achar, J. W. Hanneken, and T. Clarke, "Damping characteristics of a fractional oscillator," Physica A, vol. 339, no. 3-4, pp. 311-319, 2004.

[19] B. N. Narahari Achar, J. W. Hanneken, T. Enck, and T. Clarke, "Response characteristics of a fractional oscillator," Physica A, vol. 309, no. 3-4, pp. 275-288, 2002.

[20] A. Al-rabtah, V. S. Ertürk, and S. Momani, "Solutions of a fractional oscillator by using differential transform method," Computers and Mathematics with Applications, vol. 59, no. 3, pp. 1356-1362, 2010.

[21] S. C. Lim, M. Li, and L. P. Teo, "Locally self-similar fractional oscillator processes," Fluctuation and Noise Letters, vol. 7, no. 2, pp. L169-L179, 2007.

[22] S. C. Lim, M. Li, and L. P. Teo, "Langevin equation with two fractional orders," Physics Letters A, vol. 372, no. 42, pp. 6309-6320, 2008.

[23] S. C. Lim and S. V. Muniandy, "Self-similar Gaussian processes for modeling anomalous diffusion," Physical Review E, vol. 66, no. 2, Article ID 021114, pp. 021114/1-021114/14, 2002.

[24] S. C. Lim and L. P. Teo, "The fractional oscillator process with two indices," Journal of Physics A: Mathematical and Theoretical, vol. 42, no. 6, Article ID 065208, 34 pages, 2009.

[25] M. Li, "Fractal time series-a tutorial review," Mathematical Problems in Engineering, vol. 2010, Article ID 157264, 26 pages, 2010.

[26] I. Podlubny, Fractional Differential Equations, Academic Press, San Diego, Calif, USA, 1999.

[27] I. S. Gradshteyn and I. M. Ryzhik, Table of Integrals, Series, and Products, Elsevier, Singapore, 7th edition, 2007, edited by A. Jeffrey and D. Zwillinger.

[28] R. C. Dorf and R. H. Bishop, Modern Control Systems, Prentice Hall, Upper Saddle River, NJ, USA, 9th edition, 2002.

[29] H. K. Khalil, Nonlinear Systems, Prentice Hall, Upper Saddle River, NJ, USA, 2nd edition, 2002.

[30] M. Li and W. Zhao, "Representation of a stochastic traffic bound," IEEE Transactions on Parallel and Distributed Systems, vol. 21, no. 9, pp. 1368-1372, 2010.

[31] M. Li and S. C. Lim, "Power spectrum of generalized Cauchy process," Telecommunication Systems, vol. 43, no. 3-4, pp. 219-222, 2010.

[32] M. Li, "Generation of teletraffic of generalized Cauchy type," Physica Scripta, vol. 81, no. 2, Article ID 025007, 10 pages, 2010.

[33] M. Li and S. C. Lim, "Modeling network traffic using generalized Cauchy process," Physica A, vol. 387, no. 11, pp. 2584-2594, 2008.

[34] S. C. Lim and M. Li, "A generalized Cauchy process and its application to relaxation phenomena," Journal of Physics A: Mathematical and General, vol. 39, no. 12, pp. 2935-2951, 2006.

[35] M. Li, "Modeling autocorrelation functions of long-range dependent teletraffic series based on optimal approximation in Hilbert space-A further study," Applied Mathematical Modelling, vol. 31, no. 3, pp. 625-631, 2007.

[36] S. Y. Chen, Y. F. Li, and J. Zhang, "Vision processing for realtime 3-D data acquisition based on coded structured light," IEEE Transactions on Image Processing, vol. 17, no. 2, pp. 167-176, 2008.

[37] S. Y. Chen, Y. F. Li, Q. Guan, and G. Xiao, "Real-time three-dimensional surface measurement by color encoded light projection," Applied Physics Letters, vol. 89, no. 11, Article ID 111108, 2006.

[38] E. G. Bakhoum and C. Toma, "Mathematical transform of traveling-wave equations and phase aspects of quantum interaction," Mathematical Problems in Engineering, vol. 2010, Article ID 695208, 15 pages, 2010. 
[39] C. Cattani, "Harmonic wavelet approximation of random, fractal and high frequency signals," Telecommunication Systems, vol. 43, no. 3-4, pp. 207-217, 2010.

[40] O. M. Abuzeid, A. N. Al-Rabadi, and H. S. Alkhaldi, "Fractal geometry-based hypergeometric time series solution to the hereditary thermal creep model for the contact of rough surfaces using the Kelvin-Voigt medium," Mathematical Problems in Engineering, vol. 2010, Article ID 652306, 22 pages, 2010.

[41] C. Cattani, "Fractals and hidden symmetries in DNA," Mathematical Problems in Engineering, vol. 2010, Article ID 507056, 31 pages, 2010.

[42] J. Chen, C. Hu, and Z. Ji, "An improved ARED algorithm for congestion control of network transmission," Mathematical Problems in Engineering, vol. 2010, Article ID 329035, 14 pages, 2010.

[43] E. G. Bakhoum and C. Toma, "Dynamical aspects of macroscopic and quantum transitions due to coherence function and time series events," Mathematical Problems in Engineering, vol. 2010, Article ID 428903, 13 pages, 2010. 


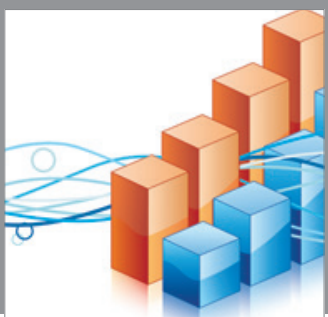

Advances in

Operations Research

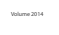

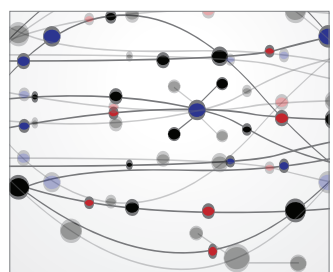

\section{The Scientific} World Journal
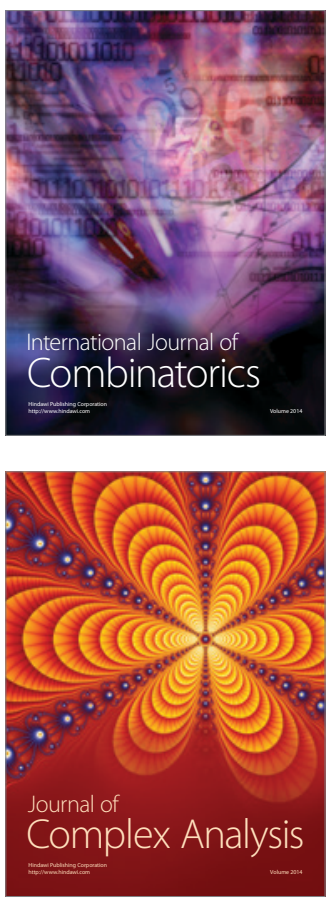

International Journal of

Mathematics and

Mathematical

Sciences
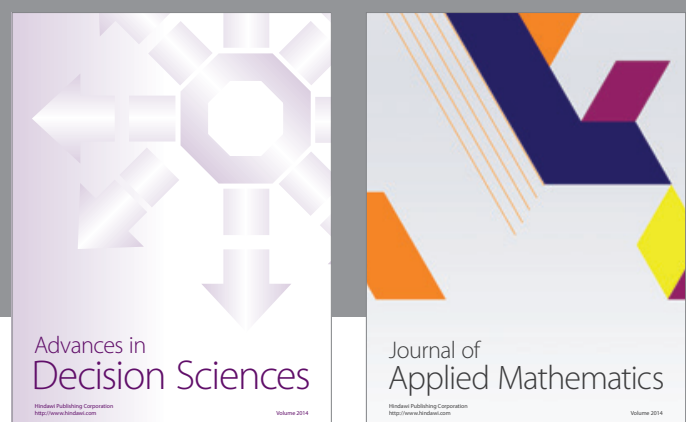

Journal of

Applied Mathematics
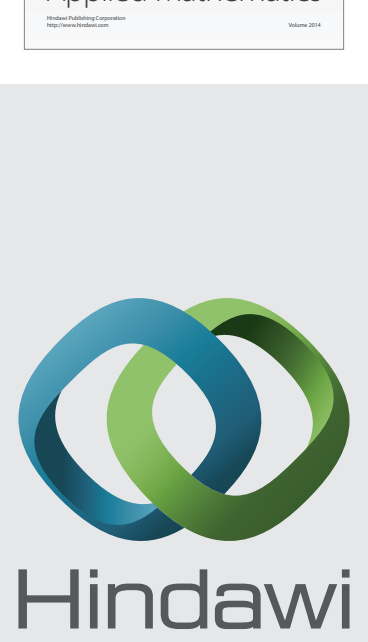

Submit your manuscripts at http://www.hindawi.com
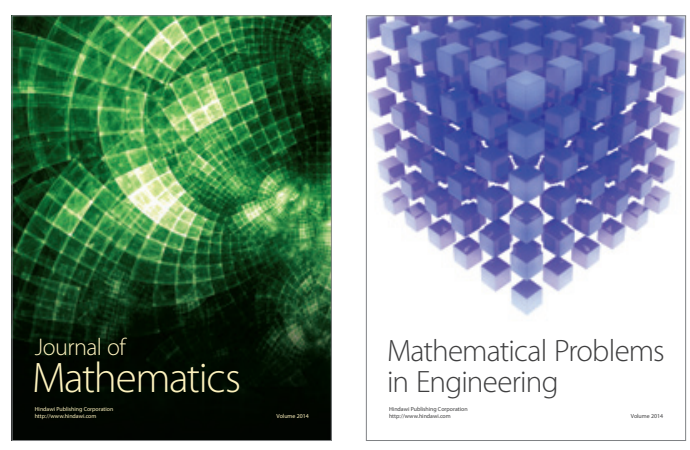

Mathematical Problems in Engineering
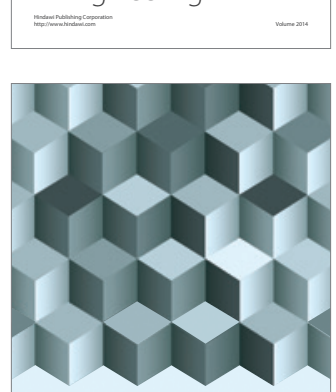

Journal of

Function Spaces
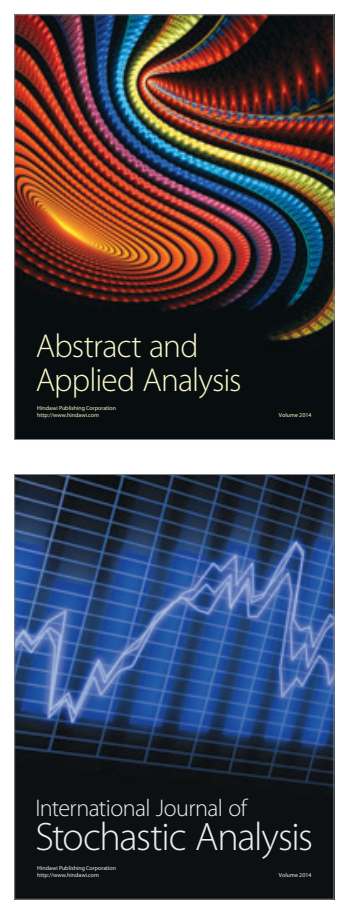

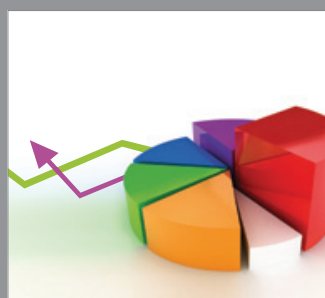

ournal of

Probability and Statistics

Promensencen
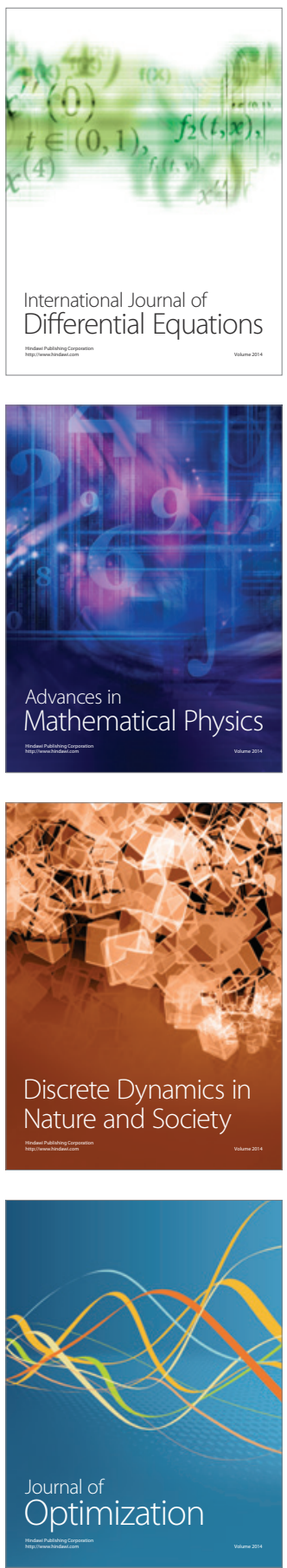